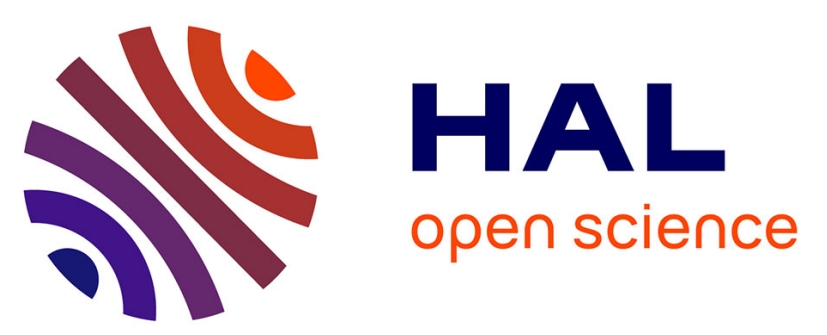

\title{
DESTRUCTION OF ANTIFERROMAGNETISM BY VACANCIES FOR THE HUBBARD AND ANDERSON LATTICE MODELS
}

\author{
C. Bastide, C. Lacroix, A. da Rosa Simões
}

\section{> To cite this version:}

C. Bastide, C. Lacroix, A. da Rosa Simões. DESTRUCTION OF ANTIFERROMAGNETISM BY VACANCIES FOR THE HUBBARD AND ANDERSON LATTICE MODELS. Journal de Physique Colloques, 1988, 49 (C8), pp.C8-715-C8-716. 10.1051/jphyscol:19888324 • jpa-00228499

\section{HAL Id: jpa-00228499 https://hal.science/jpa-00228499}

Submitted on 1 Jan 1988

HAL is a multi-disciplinary open access archive for the deposit and dissemination of scientific research documents, whether they are published or not. The documents may come from teaching and research institutions in France or abroad, or from public or private research centers.
L'archive ouverte pluridisciplinaire HAL, est destinée au dépôt et à la diffusion de documents scientifiques de niveau recherche, publiés ou non, émanant des établissements d'enseignement et de recherche français ou étrangers, des laboratoires publics ou privés. 


\title{
DESTRUCTION OF ANTIFERROMAGNETISM BY VACANCIES FOR THE HUBBARD AND ANDERSON LATTICE MODELS
}

\author{
C. Bastide, C. Lacroix and A. da Rosa Simões ${ }^{1}$ \\ Laboratoire Louis Néel, C.N.R.S., 166X, 38042 Grenoble Cedex, France
}

\begin{abstract}
We study the itinerant antiferromagnetism of fermions with hard-core repulsion, like in the weak hopping Anderson lattice or the strong $U$ Hubbard Hamiltonian. It is found that the critical temperature $T_{\mathrm{N}}$ decreases very quickly when vacancies are injected. The effect of the dynamic three-sites magnetic interaction is studied.
\end{abstract}

We study the depression of the Néel temperature for fermions with hard-core repulsion when the electronic density deviates from half-filling. Such strong local repulsions are believed to play an important role both in the heavy fermions compounds and in the high$T_{\text {c }}$ cuprate materials. The strong- $U$ single band Hubbard model and the weak hopping Anderson lattice model [1] can both be described by an effective hamiltonian involving only one kind of particles with infinite on-site repulsion and nearest-neighbour two and threesites second-order interactions. We will keep only the magnetic interactions:

$$
H_{\mathrm{eff}}=\sum_{i j \sigma} t b_{i \sigma}^{+} b_{j \sigma}-\frac{1}{2} \sum_{i j} I \mathbf{S}_{i} . \mathbf{S}_{j}-\sum_{i j l \ell} I^{\prime} \mathbf{S}_{i \ell} \cdot \mathbf{S}_{j}
$$

where $b_{i \sigma}=\left(1-a_{i-\sigma}^{+} a_{i-\sigma}\right) a_{i \sigma}, \mathbf{S}_{i}$ and $\mathbf{S}_{i \ell}$ are expressed with the $b_{i \sigma}$ as usual, $i j$ and $j \ell$ are nearest neighbours sites.

We will estimate the Neel temperature for antiferromagnetism by the difference of energy at zero temperature between the non-magnetic state and the one with a staggered magnetization $m$. The value of this antiferromagnetic magnetization will be found by minimizing the energy $\left(H_{\text {eff }}\right)$ with respect to $m$.

We consider a lattice with no frustration. We approximate the energy coming from the two last terms of (1) by:

$$
-\frac{1}{2} \sum_{i j} I\left\langle S_{i}^{z}\right\rangle\left\langle S_{j}^{z}\right\rangle-\sum_{i j \ell} I^{\prime}\left\langle S_{i \ell}^{z}\right\rangle\left\langle S_{j}^{z}\right\rangle
$$

which is supposed to be the main contribution to the $m$-dependent part, and where nearest neighbour magnetic correlations are neglected. This approximation which gives the mean-field result in the localized limit is hoped to be valid at least in three dimensions.

We describe the motion of the particles by a method which gives the good variation of the kinetic energy as a function of the staggered magnetization. Some works are related to this question [2], but we use here a very simple description: The Hubbard's I approximation [3] contains the main point necessary to our purpose which is the reduction of the bandwidth with increasing magnetization. The equation of motion for the Green's function $G_{i m \sigma}=\left\langle\left\langle b_{i \sigma}, b_{m \sigma}^{+}\right\rangle\right\rangle$is in this approximation:

$$
\omega G_{i m \sigma}=\left\langle 1-n_{i-\sigma}\right\rangle+\left\langle 1-n_{i-\sigma}\right\rangle \sum_{\ell} t_{i \ell} G_{\ell m \sigma} .
$$

We impose a given magnetization $m=$ $\sum_{\sigma} \sigma \alpha\left\langle b_{i_{\alpha} \sigma}^{+} b_{i_{\alpha} \sigma}\right\rangle$ where $\alpha= \pm 1$ is an index for the two sublattices. Note that we have not included the effect of the interactions $I$ and $I^{\prime}$ in the equation of motion (3) because we just. want to calculate $\left\langle b_{i \sigma}^{+} b_{j \sigma}\right\rangle$ (and $\left\langle b_{i \sigma}^{+} b_{\ell \sigma}\right\rangle$ for the kinetic magnetization $\left.\left\langle S_{i \ell}^{z}\right\rangle\right)$ which is governed by the first term of $H_{\text {eff }}$ the hopping amplitude $t$ - which is at least an order of magnitude larger than $I$ and $I^{\prime}$. These second order interactions nevertheless come into play indirectly through the imposed magnetization $m$. The equation (3)is easily solved introducing two propagators (intraand inter-sublattice):

$$
\begin{gathered}
G_{k \sigma}^{\alpha \alpha}=\left(1-\frac{n}{2}+\sigma \alpha \frac{m}{2}\right) \frac{\omega}{\omega^{2}-E_{k}^{2}} \\
G_{k \sigma}^{-\alpha \alpha}=\left(\left(1-\frac{n}{2}\right)^{2}-\frac{m^{2}}{4}\right) \frac{\varepsilon_{k}}{\omega^{2}-E_{k}^{2}}
\end{gathered}
$$

where $n$ is the number of effective particles per site in the effective Hamiltonian (1), and:

$$
E_{k}=\varepsilon_{k} \sqrt{\left(1-\frac{n}{2}\right)^{2}-\frac{m^{2}}{4}}
$$

The reductive factor (5) for the bandwidth is easily understood: The Hubbard's approximation means the effective hopping of a $\sigma$-electron to site $i$ is reduced by

\footnotetext{
${ }^{1}$ Permanent address: Instituto de Fisica, UFRGS 9000 Porto Alegre, Rio Grande do Sul, Brazil.
} 
the factor $\left\langle 1-n_{i-\sigma}\right\rangle$ which is alternatively $1-n / 2-$ $m / 2$ and $1-n / 2+m / 2$ when the $\sigma$-electron moves through the lattice. So the mean effective hopping must verify:

$$
t_{\text {eff }}^{2}=\left(1-\frac{n}{2}-\frac{m}{2}\right) t\left(1-\frac{n}{2}+\frac{m}{2}\right) t
$$

which gives the same reductive factor (5).

The electronic density is easily calculated from (4) where $f$ is the Fermi-Dirac function:

$$
\frac{n}{2-n}=\frac{1}{N} \sum_{k} f\left(E_{k}\right) \quad f(x)=\left(\mathrm{e}^{\beta(x-\mu)}+1\right)^{-1} .
$$

The kinetic energy (first term of (1)) is easily obtained from the Green's function:

$$
K=K_{0} \sqrt{1-\frac{m^{2}}{(2-n)^{2}}}
$$

where $K_{0}$ is the kinetic energy of the non-magnetic state. Similarly, the kinetic magnetization $\left\langle S_{i \ell}^{z}\right\rangle$ is derived, and then the contribution of $I^{\prime}$ to the energy per site:

$$
\frac{I^{\prime}}{4} m^{2}\left[-z \frac{n}{2-n}+\frac{1}{N} \sum_{k}\left(\frac{\varepsilon_{k}}{t}\right)^{2} f\left(E_{k}\right)\right]
$$

where $z$ is the number of nearest neighbour. One can show that the expression (9) changes of sign at $n=$ 2/3. A positive $I^{\prime}$ favors antiferromagnetism for $n>$ $2 / 3$ and favors ferromagnetism for $n<2 / 3$. This is due to the sign of $\left\langle b_{i \sigma}^{+} b_{\ell \sigma}\right\rangle$ which depends upon the electronic density. When $\delta \ll 1$, the energy (9) can be written:

$$
-\frac{1}{2} z(z-1) \delta I^{\prime} m^{2}
$$

and the total energy per site is:

$$
E=K_{0} \sqrt{1-\frac{m^{2}}{(1+\delta)^{2}}}+\frac{z}{8}\left(I-4(z-1) \delta I^{\prime}\right) m^{2} .
$$

By minimizing $E$ with respect to $m$, we find the critical value $\delta_{c}$ for the number of vacancies at which antiferromagnetism disapperas:

$$
\delta_{c}=-\frac{I / 4}{2|t|-(z-1) I^{\prime}} .
$$

We recall that both in the Anderson lattice and in the Hubbard model, $I$ is negative. A negative $I^{\prime}$ like in the Hubbard model reduces $\delta_{c}$. But in the validity domain of the perturbation expansion in $t$ which leads to (1), the second order interactions $I$ and $I^{\prime}$ are far smaller than $t$, and the effect of $I^{\prime}$ is very small and can be neglected. The magnetization as a function of $\delta$ is:

$$
m=\sqrt{1-64 \delta^{2} \frac{t^{2}}{I^{2}}}
$$

and the difference of energy between the antiferromagnetic and non-magnetic state is:

$$
\Delta E=-\frac{8 z t^{2}}{I}\left(\delta+\frac{I}{8|t|}\right)^{2} .
$$

These two quantities are shown in figure 1. The critical temperature $T_{\mathrm{N}}$ is expected to have the same shape as $\Delta E$ and one can notice the upward curvature of $\Delta E(\delta)$ and the difference of shape with $m(\delta) . \delta_{\mathrm{c}}$ is a small value; for example in the Hubbard model where $I=-4 t^{2} / U$ :

$$
\delta_{\mathrm{c}} \simeq-I / 8|t|=|t| / 2 U .
$$

This quick destruction of the antiferromagnetic phase is due to the kinetic energy of the vacancies which is larger in the non-magnetic state than in the magnetic one. Yokoyama and Shiba [4] have obtained with a Gutzwiller variational technique a smaller decrease by a factor of two (a larger $\delta_{c}$ than (15)). The three-sites magnetic interaction which needs a sufficient amount of vacancies, do not play an important role for such electronic densities, this being very different from the work of Inui, Doniach and Gabay [5].

For the Anderson lattice, using the parameters calculated in [1], antiferromagnetism is then found only in the vicinity of $n=1$, and leaves the superconductivity appear for $n>1[6]$.

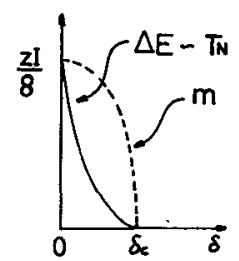

Fig. 1. - Destruction of the antiferromagnetic phase as a function of the number of vacancies $\delta=1-n$. The staggered magnetization $m$ goes from 1 to zero.

[1] Hirsch, J. E., Phys. Rev. Lett. 54 (1985) 1317; Bastide, C., Lacroix, C., Europhys. Lett. 4 (1987) 935; J. Phys. C. 21 (1988) 3557.

[2] Bulaevskii, L. N., Khomskii, D. I., Sov. Phys. JETP 25 (1967) 1067;

Brinkmann, W. F., Rice, T. M., Phys. Rev. B 2 (1970) 1324;

Joynt, R., Phys. Rev. B 37 (1988) 7979.

[3] Hubbard, J., Proc. R. Soc. A 276 (1963) 238.

[4] Yokoyama, H., Shiba, H., J. Phys. Soc. Jpn 56 (1987) 3570.

[5] Inui, M., Doniach, S., Gabay, M., preprint.

[6] Da Rosa Simões, A., Lacroix, C., Bastide, C., Sixth Int. Conf. on Crystal-Field Effects and Heavy Fermion Physics (July 1988) Frankfurt. 